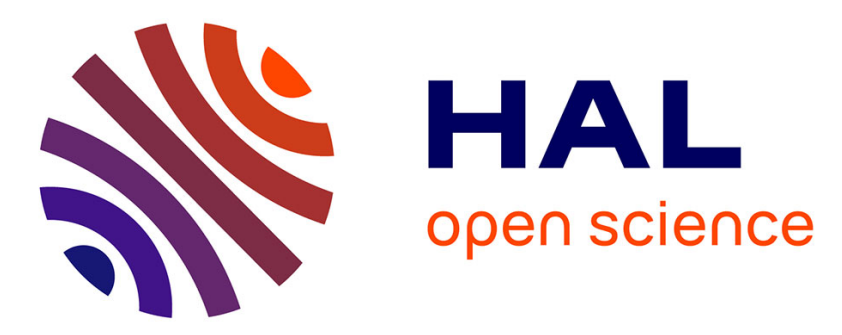

\title{
Growth, Spectroscopic and Photorefractive investigation of vanadium-doped cadmium telluride
}

Jean-Claude Launay, V. Mazoyer, M. Tapiero, Jean-Paul Zielinger, Z. Guellil, Philippe Delaye, Gérald Roosen

\section{- To cite this version:}

Jean-Claude Launay, V. Mazoyer, M. Tapiero, Jean-Paul Zielinger, Z. Guellil, et al.. Growth, Spectroscopic and Photorefractive investigation of vanadium-doped cadmium telluride. Applied physics. A, Materials science \& processing, 1992, 55, pp.33-40. 10.1007/BF00324599 . hal-00677564

HAL Id: hal-00677564

https://hal-iogs.archives-ouvertes.fr/hal-00677564

Submitted on 8 Mar 2012

HAL is a multi-disciplinary open access archive for the deposit and dissemination of scientific research documents, whether they are published or not. The documents may come from teaching and research institutions in France or abroad, or from public or private research centers.
L'archive ouverte pluridisciplinaire HAL, est destinée au dépôt et à la diffusion de documents scientifiques de niveau recherche, publiés ou non, émanant des établissements d'enseignement et de recherche français ou étrangers, des laboratoires publics ou privés. 


\title{
Growth, Spectroscopic and Photorefractive investigation of vanadium-doped cadmium telluride
}

\author{
J.C. Launay 1,2, V. Mazoyer1,
}

1) Pôle de Recherche Aquitain pour les matériaux dans l'espace (Prame), B.P. 11, 33165 Saint Médard en Jalles Cedex, France

2) Laboratoire de Chimie du Solide du Centre National de la Recherche Scientifique, Université de Bordeaux I, 33405 Talence Cedex, France

\section{Tapiero ${ }^{3}$, J.P. Zielinger ${ }^{3}$, Z. Guellil ${ }^{3}$,}

3) Institut de Physique et Chimie des Matériaux de Strasbourg, Unité Mixte 380046

CNRS-ULP-EHICS, 5 rue de l'Université, 67084 Strasbourg Cedex, France

\author{
Ph. Delaye ${ }^{4}$, G. Roosen 4 \\ 4) Institut d'Optique Théorique et Appliquée, Unité Associée au Centre National de la \\ Recherche Scientifique, Centre Scientifique d'Orsay, \\ B.P. 147, 91403 Orsay Cedex, France. \\ Tel: 1-69416855, Fax: 1-69413192
}

\begin{abstract}
We present new results on the growth of semi-insulating vanadium-doped cadmium telluride crystals and their characterizations by different optical techniques such as photoinduced current transient spectroscopy, absorption, photoconductivity spectra and photorefractive wave mixing. Our joint research program aims at developping optimized crystals for efficient optical processing in the near infrared through the photorefractive effect.
\end{abstract}

PACS: $42.65,42.70,72.40$. 


\title{
Growth, Spectroscopic and Photorefractive investigation of vanadium-doped cadmium telluride
}

\author{
J.C. Launay1,2, V. Mazoyer1, \\ 1) Pôle de Recherche Aquitain pour les matériaux dans l'espace (Prame), B.P. 11, \\ 33165 Saint Médard en Jalles Cedex, France \\ 2) Laboratoire de Chimie du Solide du Centre National de la Recherche Scientifique, \\ Université de Bordeaux I, 33405 Talence Cedex, France \\ M. Tapiero ${ }^{3}$, J.P. Zielinger ${ }^{3}$, Z. Guellil ${ }^{3}$, \\ 3) Institut de Physique et Chimie des Matériaux de Strasbourg, Unité Mixte 380046 \\ CNRS-ULP-EHICS, 5 rue de l'Université, \\ 67084 Strasbourg Cedex, France \\ Ph. Delaye ${ }^{4}$, G. Roosen 4 \\ 4) Institut d'Optique Théorique et Appliquée, Unité Associée au Centre National de la \\ Recherche Scientifique, Centre Scientifique d'Orsay, \\ B.P. 147, 91403 Orsay Cedex, France.
}

\section{Introduction}

The investigation of photorefractive semiconductors is strongly motivated by the compatibility of their operating wavelengths with semiconductor lasers and with fiber optics communications.

For a better understanding of the complex processes occuring in photorefractive materials, a variety of research area are now involved including not only nonlinear optical properties but also crystal growth, spectroscopy, etc.... In this context, the determination of deep trapping levels that directly affect the photorefractive properties is of prime importance. 
For a long time, it was generally believed that only the dominant deepest levels were involved. However, since the advent of experiments conducted with pulsed high energy nanosecond and picosecond lasers, many new interesting phenomena have been discovered. Clearly, in the pulse regime, shallower levels play also an important role $(1,2)$. Any defect, provided that its concentration is of the order of $10^{15} \mathrm{~cm}^{-3}$, may be concerned. Therefore, a complete knowledge of the imperfection identity, thermal and photoionization energies, capture cross sections and concentration is desirable for optimum control of the material parameters to meet device requirements.

The introduction of transition metals (TM) into II-VI compounds usually causes considerable changes in the electrical, optical and photoelectrical properties due to charge compensation and additionnal extrinsic absorption and photoconductivity.

Some years ago, vanadium (V) has been identified as a suitable dopant for producing appropriate deep levels in cadmium telluride, permitting the generation of the photorefractive effect $(3)$. The corresponding energy level is expected to act as a donor and to lie at $\mathrm{E}_{\mathrm{c}}-0.75 \mathrm{eV}$, i.e. in the middle of the band gap ${ }^{(4)}$. This is a location very favourable for the presence of the dopant in both unionized (0) and ionized (+) states, the unionized centers providing the photoinduced carriers.

Provided that the lattice relaxation of vanadium in CdTe is not too high, as can be expected from the pronounced covalent character of the compound, the onset of optical absorption and photorefractivity is expected at $1.7-1.5 \mathrm{eV}$. The relatively high thermal ionization energy prevents immediate reemission of the trapped charges.

Recently, photorefractive energy transfer at $1.5 \mu \mathrm{m}$ has been reported in CdTe: $\mathrm{V}^{(5)}$ showing that indeed this material is a good candidate for optical information processing using low power (mW) lasers.

Unfortunately, very little is known about vanadium in CdTe. Most of the informations were obtained in connection with the previously mentioned investigations of photorefractive properties.

Therefore, in France, we have recently started a joint research program with the aim of obtaining information on the influence of isocharge $\mathrm{V}$ impurities on the characteristics 
of doped CdTe crystals and then of optimizing them regarding the key photorefractive parameters.

We here present our first results. We have grown and vanadium doped good quality semi-insulating cadmium telluride crystals and have conducted spectroscopic and photorefractive characterizations on the same samples.

\section{Crystal growth}

\subsection{Experimental conditions}

The growth conditions were established to satisfy the following criteria:

- single crystallinity of the ingot,

- low dislocation density and no tellurium precipitation.

V-doped CdTe crystals were grown using the modified Bridgman technique from CdTe (6-9's grade) in evacuated (10-6 torr) graphitized quartz ampoules (to minimize nucleation sites). The purity and concentration of vanadium were respectively at least 5-9's grade and $10^{19}-10^{20}$ at. $\mathrm{cm}^{-3}$. Crystals were grown with the dopant either added either compensated to the melt.

The ampoule took place in a vertical furnace under the following conditions: heating to $1125^{\circ} \mathrm{C}$ at a rate of $50^{\circ} \cdot \mathrm{h}^{-1}$ and maintaining this temperature for $20 \mathrm{~h}$. Single crystals were grown at a rate of $6 \mathrm{~mm} \cdot \mathrm{day}^{-1}$ in a typical gradient of $5^{\circ} . \mathrm{cm}^{-1}$. Experimental conditions take into account the low thermal conductivity of CdTe. The single crystal volume is routinely greater than $80 \%$.

\subsection{Chemical composition}

The distribution of $\mathrm{Cd}$, Te and $\mathrm{V}$, as observed from microprobe elementary mapping is homogeneous. The deviation of nominal composition inferred from the microprobe 
analysis is below the limit of detection sensitivity of this technique. No precipitate of Te was observed under IR microscopic examination.

Substitutional $\mathrm{V}$ is paramagnetic in its neutral charge state $\mathrm{V}^{2+}$, corresponding to a $3 \mathrm{~d}^{3}$ configuration. At room temperature, the ESR signal of $\mathrm{V}^{2+}$ is not observed due to short relaxation time or electronic delocalization. When cooled down to liquid helium temperature, delocalization might be stopped and we thus should observe the ESR signal $(6)$.

\subsection{Lattice parameter}

The lattice parameter (a) determined by X-ray diffraction is $0.6453 \mathrm{~nm}$. This is smaller than the value $(\mathrm{a}=0.6484 \mathrm{~nm})$ reported for CdTe in ASTM data. The V atom $(\mathrm{Z}=23)$ is smaller than the $\mathrm{Cd}$ atom $(\mathrm{Z}=48)$. The screening effect of the electron shells is smaller in the V case than in the Cd one. This leads the V-Te bond length to be shorter than the Cd-Te one $(0.2794 \mathrm{~nm})$.

\section{$\underline{2.4 \text { Microhardness }}$}

Microhardness tests were performed at room temperature using a Vickers microscope mounted indentor system SHIMADZU HMV-2000. Hardness data provide a good general impression of the mechanical properties of the materials and of their bounding strength. Many intents were made on each slice, with a rotation between them. This excludes any hardness variation due to cristallographic anisotropy of the average values that were obtained.

From $\mathrm{H}_{\mathrm{V}}=1.854 \mathrm{~F} / \mathrm{D}^{2}$ relation $(\mathrm{F}=$ test charge in $\mathrm{Kg}, \mathrm{D}=$ length of the indent $)$ the microhardness is the same along axis and radial ingot and similar to the CdTe one (47.5 +/- $0.5 \mathrm{Kg} \cdot \mathrm{mm}^{-2}$ ). No significant microhardness dependence appears with $\mathrm{V}$ dopant even at $10^{20}$ at. $\mathrm{cm}^{-3}$ concentration. 


\subsection{Resistivity measurement}

Electrical resistivity of the sample were measured by a conventional two point technic with silver contacts. The contact resistance was negligible. Actually, contact problems are less critical for high resistivity materials than for semiconductors. Measurements were carried out from room temperature to $400 \mathrm{~K}$ and then down to room temperature. Resistivity is about $5 \times 10^{10}$ and $5 \times 10^{8} \Omega . \mathrm{cm}$ for CdTe: $\mathrm{V}$ with $\mathrm{V}$ concentrations of $10^{20}$ (sample 6B) and $10^{19}$ at. $\mathrm{cm}^{-3}$ (sample $1 \mathrm{H}$ ). We notice that the results are reproducible after rising and cooling the temperature. These crystals thus present the required resistivity for the generation of optical nonlinearities through the photorefractive effect with low power lasers.

\section{Spectroscopic characterization.}

It must be pointed out that semi-insulating CdTe can also be grown by the travelling heater method (THM) using tellurium solvant with chlorine ${ }^{(7)}$. However, the electrical resistivity does not exceed $10^{8} \Omega . \mathrm{cm}$. In this type of material a large number of different trap species have been detected by using Thermally Stimulated Current (TSC) and PhotoInduced Transient Spectroscopy (PICTS) techniques ${ }^{(7,8)}$. The spectral resolution was not sufficient to allow a complete and precise determination of the trap parameters. Nevertheless, a few centers could be identified and characterized. Two levels may be efficient as traps in room temperature operation respectively at $\mathrm{E}_{\mathrm{C}}$ $0.58 \mathrm{eV}$ (interstitial Cd or Te vacancy) and $\mathrm{EC}-0.7 \mathrm{eV}$ (double negatively charged $\mathrm{Cd}$ vacancy $\left.\mathrm{V}_{\mathrm{Cd}^{-}}{ }^{-}\right)$. On the other hand the single charged vacancy $\left(\mathrm{V}_{\mathrm{Cd}^{-}}{ }^{-}\right.$, state - located at $\mathrm{EV}+0.4 \mathrm{eV}$ in $\mathrm{CdTe}$, is known to play an important role as a compensating acceptor and as a recombination center.

Introduction of TM may cause rather complex compensation phenomena between the various types of native defects. Actually only slight changes in the edge luminescence spectra were observed, as long as the $\mathrm{V}$ concentration was lower than $10{ }^{19} \mathrm{~cm}^{-3(9)}$. 
However a further increase in V-concentration $\left(10^{20} \mathrm{~cm}^{-3}\right)$ revealed new D-A transitions. The following interpretation was proposed: introduction of $\mathrm{V}$ at increasing concentrations first results in the filling of $\mathrm{Cd}$ vacancies and finally in the occupation of interstitial sites, giving rise to a new donor level.

As pointed out before, the determination of deep level spectra is one of the important problems. We report a set of first results obtained by an improved PICTS technique (10)

\subsection{Experimental method.}

PICTS is a transient photoconductivity, computer based, measuring technique. The current transient, including both rise and decay, induced by a square light pulse in a biased sample, is recorded every 1 or $2 \mathrm{~K}$, during a temperature scan (usually from 80 $\mathrm{K}$ up to $500 \mathrm{~K}$, or vice versa). Sufficient time is provided for allowing the photocurrent to reach saturation and after that to decay to zero. A slow decay (typically $10^{-3}$ s up to $10 \mathrm{~s}$ ) indicates thermally stimulated emission from traps (Fig. 1).

The slow component has a complex thermal behaviour: both the amplitude, the shape and the decay time vary. Three types of numerical processing of the stored data allow us to extract the parameters of the traps: the apparent thermal ionization energy $E_{t}$ and capture cross section $\mathrm{S}_{\mathrm{t}}$, the concentration $\mathrm{N}_{\mathrm{t}}$ for the dominant species and perhaps also the type $n$ or $\mathrm{p}^{(10)}$.

In order to stress the difference between the three data treatments and hence the signification of the curves presented hereafter and also to emphasize the improvement of our procedure with respect to similar measurements bearing the same initials, we briefly recall the principles of the calculation.

For sake of simplicity we consider the single trap case. The analysis further assumes that the thermally released carriers are not retrapped, but are neutralized by carriers of opposite sign localized on deeper lying levels. The trapped charge $n_{t}(t)$ then decays exponentially according to:

$$
n_{t}(t)=N_{t} \exp \left(-t / \tau_{t}\right)
$$


The relaxation time $\tau_{t}$ is related to $E_{t}$ and $S_{t}$ :

$$
\tau_{\mathrm{t}}{ }^{-1}=\mathrm{A} \mathrm{S}_{\mathrm{t}} \mathrm{T}^{2} \exp \left(-\mathrm{E}_{\mathrm{t}} / \mathrm{kT}\right)
$$

where $\mathrm{k}$ is the Boltzmann constant and A a constant ${ }^{(7)}$.

Suppose a signal which directly reflects the decay of $\mathrm{n}_{\mathrm{t}}$ (equat.1) be recorded at various temperatures. As $\tau_{t}$ stongly depends on $T$, the shape of the transient changes as shown in Fig.2. If the signal is sampled at two fixed delay times $t_{1}$ and $t_{2}$, the difference $\Delta \mathrm{n}=$ $\mathrm{n}_{\mathrm{t}}\left(\mathrm{t}_{1}\right)-\mathrm{n}_{\mathrm{t}}\left(\mathrm{t}_{2}\right)$ obviously goes through a maximum at a temperature $\mathrm{T}_{\mathrm{m}}$ where $\tau_{\mathrm{t}}$ takes a defined value $\tau_{m}$ which can be related mathematically to $t_{1}$ and $t_{2}$ using the condition for the maximum of the function

$$
f(\tau t)=\exp \left(-t_{1} / \tau t\right)-\exp \left(-t_{2} / \tau_{t}\right)
$$

The result is that $\tau_{\mathrm{m}}$ can be computed knowing $t_{1}$ and $t_{2}$ whereas $T_{m}$ is obtained from fig.2.

This is the key idea of PICTS. Actually the curve is plotted for several couples $\left(\mathrm{t}_{1}, \mathrm{t}_{2}\right)$, so maxima are obtained at different temperatures $\mathrm{T}_{\mathrm{m}}$. The Arrhenius plot $\log \left(\tau_{\mathrm{m}} \mathrm{T}_{\mathrm{m}}{ }^{2}\right)=\mathrm{f}\left(1 / \mathrm{T}_{\mathrm{m}}\right)$ yields $\mathrm{E}_{\mathrm{t}}$ and $\mathrm{S}_{\mathrm{t}}$ according to (2). The height of the peak provides a measure of $\mathrm{N}_{\mathrm{t}}(10)$.

In practice, however $n_{t}(t)$ is not directly accessible. But the transient current, due to the thermal release of the trapped charge, can be measured. This current is proportionnal to $\mathrm{dn}_{\mathrm{t}} / \mathrm{dt}=\mathrm{N}_{\mathrm{t}} / \tau_{\mathrm{t}} \cdot \exp \left(-\mathrm{t} / \tau_{\mathrm{t}}\right)$. On the other hand, it depends on the mobility $\mu$ and on the recombination life time $\tau$.

The current can be expressed as :

$$
\mathrm{i}(\mathrm{t})=\left\{\mathrm{B} \mu \tau \mathrm{N}_{\mathrm{t}} / \tau_{\mathrm{t}}\right\} \exp \left(-\mathrm{t} / \tau_{\mathrm{t}}\right)
$$

where B is a constant including the applied electrical field and a geometrical factor.

Usually the temperature dependence of $\mu \tau$ is neglected in $(3)^{(10)}$. The relation expressing the condition for the maximum of $g\left(\tau_{t}\right)=1 / \tau_{t}$. $\left[\exp \left(-t_{1} / \tau_{t}\right)-\exp \left(-t_{2} / \tau_{t}\right)\right]$ is thus used for the determination of $\tau_{\mathrm{m}}$. This simple but approximative conventional procedure does not yield very reliable values for the trap parameters.

A better alternative is to divide the double-gate signal by the photocurrent $\mathrm{i}(0)$ which is proportionnal to $\mu \tau$ (Fig.1), a procedure which allows to eliminate, in a first 
approximation the $\mu \tau$ product in (3). This method is of special interest for the determination of the trap concentrations (10). Actually it introduces another temperature dependant factor, i.e. the optical generation rate $\mathrm{G}$ of photocarriers. In general, however $\mathrm{G}$ can be kept fairly constant (see section 3.2 ) by properly adjusting the wavelength of the excitation.

The problem can be led back to the transient charge case (equation 1) simply by reading the current at four fixed delay times $\mathrm{t}_{0}, \mathrm{t}_{1}, \mathrm{t}_{2}, \mathrm{t}_{3}$ and plotting the function :

$$
\mathrm{Y}(\mathrm{T})=\left[\mathrm{i}\left(\mathrm{t}_{1}\right)-\mathrm{i}\left(\mathrm{t}_{2}\right)\right] /\left[\mathrm{i}\left(\mathrm{t}_{0}\right)-\mathrm{i}\left(\mathrm{t}_{3}\right)\right]
$$

It must be pointed out that this "four-gate" treatment eliminates the pre-exponential factor in (3), including $N_{t}$. $Y(T)$ shows a maximum provided that $t_{0}<t_{1}<t_{2}<<t_{3}(10)$. The four-gate data processing is a very sensitive method with high spectral resolution. It represents a considerable improvement for the determination of the trap signatures $\left(\mathrm{E}_{\mathrm{t}}, \mathrm{S}_{\mathrm{t}}\right)$.

\subsection{Results and discussion.}

\section{A. Electrical conductivity:}

The electrical conductivity versus $\mathrm{T}$ has been measured on two samples $1 \mathrm{H}$ and $6 \mathrm{~B}$, the second one beeing stronger doped $\left(\sim 10^{20} \mathrm{~cm}^{-3}\right)$ than the first one $\left(<10^{19} \mathrm{~cm}^{-3}\right)$. In a temperature range extending from somewhat below $300 \mathrm{~K}$ up to $400 \mathrm{~K}$, nearly the same activation energy was found with both samples : $0.76 \mathrm{eV}$ for sample $1 \mathrm{H}$ and 0.72 $\mathrm{eV}$ for sample 6B. This result confirms that the dark Fermi level is pinned by a deep level lying close to the middle of the bandgap. 
B. Spectral response of the photoconductivity:

Figures 3 shows the normalized spectral responses of samples $1 \mathrm{H}$ and $6 \mathrm{~B}$ respectively. The peak at roughly $1.5 \mathrm{eV}$ corresponds to band to band transitions.

In the case of sample $1 \mathrm{H}$ the extrinsic photoconductivity drops sharply for photon energies $\mathrm{h} v<1.1 \mathrm{eV}$. This result fits well with the measured absorption spectrum (section3) as well as with the photoconductivity curve of ref. 3 and their corresponding absorption spectrum. It also agrees with the absorption band of undoped $\mathrm{CdTe}^{(11)}$. We therefore conclude that, at low doping rate, $\mathrm{V}$ is not optically active.

The photoconductivity band for sample $6 \mathrm{~B}$ on the contrary extends up to $1.7 \mu \mathrm{m}$. In this sample, photorefractivity should be observable at $1.3-1.5 \mu \mathrm{m}$. As stated by P.I.Babii et al. ${ }^{(9)}$ additionnal deep states can only be introduced by heavy doping.

\section{Determination of trap parameters:}

In order to obtain reliable results the PICTS technique requires optimal experimental conditions which depend on the material ${ }^{(10)}$. Concerning CdTe, we shall come back to this problem in a forthcoming paper when more complete results on a larger number of crystals will be available. We describe here only a few of the experimental conditions which are important for the understanding and discussion of the results. In this paper we present a list of the detected traps with a first estimation of their parameters.

Most of the presented results concern sample $1 \mathrm{H}$. The transients were recorded while the temperature was scanned between $80 \mathrm{~K}$ and $330 \mathrm{~K}$. A first experiment was performed the sample beeing excited with photons of energy close to the maximum of the photoconductivity spectrum.

The maximum photoconductivity near the absorption edge usually occurs for that wavelength $\lambda=\lambda$ max corresponding to an absorption constant $\alpha_{\max }$ approximately equal to the reciprocal of the thickness $d$ of the crystal, i.e. where (1-1/e) of the incident 
radiation is absorbed. For $\lambda>\lambda_{\max }$, the light is completely absorbed and the current should be constant. In fact it decreases due to the shorter recombination lifetime of photocarriers generated in the vicinity of the surface and the strongly increasing absorption.

During the temperature scan $\lambda_{\max }$ was continiously adjusted to achieve maximum photoresponse. The optical generation rate $\mathrm{G}$ of photocarriers:

$$
\mathrm{G}=\alpha_{\max } \Phi_{\mathrm{O}}=\Phi_{\mathrm{O}} / \mathrm{d} \quad\left(\Phi_{\mathrm{O}} \text { is the incident photons flux }\right)
$$

can thus be considered to be temperature independent. Under these conditions the plot of the photocurrent (i(0) on Fig.1) versus temperature represents the temperature dependence of the $\mu \tau$ product. (Fig.4, curve 1)

As an example, Fig.4 also shows a double-gate (D.G) spectrum in the range 80 - 300 K. Some poorly resolved structures are visible. They are clearly superimposed on a background which reflects the shape of the $\mu \tau=f(T)$ curve. The normalized doublegate (N.D.G) spectrum brings some improvement (Fig.5). But the four-gate spectrum (F.G) undeniably provides the best spectral resolution. Six peaks appear in the considered temperature interval, but there is at least another one below $80 \mathrm{~K}$ and another one above $370 \mathrm{~K}$.

Each of the F.G. peak has been analysed separately, according to the above defined procedure which yields an Arrhenius plot from which $\mathrm{E}_{\mathrm{t}}$ and $\mathrm{S}_{\mathrm{t}}$ are obtained (Fig.6). The results are listed in Table 1.

In order to explore the forbiden bandgap for deeper levels the temperature should be raised up to $500 \mathrm{~K}$. The point is that above $350 \mathrm{~K}$ the dark current begins to increase more and more rapidly, making the differential measurements difficult. Nevertheless a first attempt has clearly revealed the presence of an additional peak at about $380 \mathrm{~K}$ corresponding to a trap depth $\mathrm{Et}=0.7 \mathrm{eV}$.

It is out of scope of this paper to discuss in detail the results listed in table 1. However it is worthy making a few remarks :

- The values are reproducible when the excitation conditions are changed. 
- Part of the detected traps are identical with those quoted in the introduction, but additional peaks are revealed which were not resolved in TSC or conventional PICTS (8). In all probability, the same species are always simultaneously present in the material, whatever the growth technique used. However their concentration may differ from sample to sample, or at least depend on preparation, conductivity, doping, etc...

Our method will allow to analyse this point ${ }^{(10)}$.

The investigation of trapping levels deeper than $\sim 0.6 \mathrm{eV}$ will be of particular interest for photorefractivity. Work will be continued in this direction.

\section{Photorefractive analysis by two wave mixing}

We here present the absorption, photoconductivity and energy transfer measurements we have conducted on sample $1 \mathrm{H}\left(\mathrm{CdTe}: \mathrm{V}\right.$ with $\mathrm{V}$ concentrations of about $10^{19} \mathrm{at}^{\mathrm{cm}} \mathrm{cm}^{-}$ 3).

\subsection{Absorption spectrum, photoconductivity and dielectric relaxation time.}

The absorption spectrum of sample $1 \mathrm{H}$ is shown in figure 7 . The shape of this spectrum is very similar to the one measured in ref. 3. However our absorption values are smaller. We obtained:

$$
\begin{aligned}
& \alpha=(2.16+/-0.05) \mathrm{cm}^{-1} \text { at } \lambda=1.06 \mu \mathrm{m}, \\
& \alpha=(1.62+/-0.05) \mathrm{cm}^{-1} \text { at } \lambda=1.32 \mu \mathrm{m}, \\
& \alpha=(1.55+/-0.05) \mathrm{cm}^{-1} \text { at } \lambda=1.55 \mu \mathrm{m} .
\end{aligned}
$$

At wavelength $\lambda=1.06 \mu \mathrm{m}$, we determined a conductivity given by:

$$
\sigma=\sigma_{\mathrm{d}}+\sigma_{\mathrm{ph}}=(1.5+0.7 \mathrm{I} 0) \cdot 10^{-9}(\Omega . \mathrm{cm})^{-1}
$$

where $\mathrm{I}_{0}$ is the incident irradiance in $\mathrm{mW} \cdot \mathrm{cm}^{-2}$. Taking the relative permittivity value $\varepsilon_{\mathrm{r}}=10.3^{(12)}$, this corresponds to a dielectric relaxation time $\tau_{\mathrm{di}}=14 \mu \mathrm{s}$ for an incident illumination $\mathrm{I}_{0}=100 \mathrm{~mW} \cdot \mathrm{cm}^{-2}$. From this value of $\sigma_{\mathrm{ph}}$, one derives an estimate of $\mu \tau$, the mobility-life time product as: 


$$
\sigma_{\mathrm{ph}}=\mu \tau \Phi \mathrm{I}_{\mathrm{a}} \mathrm{e} / \mathrm{h} v
$$

where $I_{a}$ is the absorbed intensity in the crystal, $\Phi$ the quantum efficiency, e the electron charge and hv the photon energy. The absorption coefficient of our sample being $\alpha=2 \mathrm{~cm}^{-1}$, assuming a quantum efficiency of 1 , one gets:

$$
\mu \tau=8 \cdot 10^{-7} \mathrm{~cm}^{2} \mathrm{~V}^{-1}
$$

As shown in ref. 2, this product is not affected by the presence of shallow traps that only change, in a reverse manner, $\mu$ and $\tau$, i.e., reduce the mobility and increase the recombination time by factors that characterize these shallow traps.

At wavelength $\lambda=1.32 \mu \mathrm{m}$, we determined a conductivity expressed as:

$$
\sigma=\sigma_{\mathrm{d}}+\sigma_{\mathrm{ph}}=\left(1.5+0.2 \mathrm{I}_{0}\right) \cdot 10^{-9}(\Omega . \mathrm{cm})^{-1} \text {. Considering the }
$$

absorbed energy, this correspond to nearly a 5 time drop in the photoconductivity compared to what we obtained at $\lambda=1.06 \mu \mathrm{m}$, as already seen on the photoconductivity spectra presented in section 3 .

\subsection{Effective trap density and diffusion length.}

With the set-up we previously used for characterizing $\operatorname{InP}$ and $\operatorname{GaAs}(13)$, we have investigated the photorefractive energy transfer between two waves interfering inside the crystal. At both $\lambda=1.06$ and $1.32 \mu \mathrm{m}$, the light source was a diode pumped YAG laser. The beams were polarized $+/-45^{\circ}$ respective to the crystal $(0,0,1)$ axis and were propagating along $(1,-1,0)$. The grating wave vector was along the $(1,1,0)$ direction.

Switching both beam polarizations from $+45^{\circ}$ to $-45^{\circ}$ changes the sign of the photorefractive gain and thus permits to discriminate between different sources of energy transfer (14). Our experiments show that we here get a genuine photorefractive effect.

We then rapidely studied the photorefractive gain as a function of the input intensity. Its dependence has the form:

$$
\Gamma\left(\mathrm{I}_{0}\right)=\Gamma /\left(1+\mathrm{I}_{\mathrm{d}} / \mathrm{I}_{0}\right)
$$


where $\Gamma$ is the saturated gain coefficient and $I_{d}$ is the equivalent dark irradiance, i.e., the illumination at which photoconductivity equals dark conductivity. Figure 8 demonstrates that the gain saturates at illumination values as low as $20 \mathrm{~mW} . \mathrm{cm}^{-2}$, showing that this material is indeed a good candidate for optical information processing with low power lasers. At a grating spacing $\Lambda=0.9 \mu \mathrm{m}$, one gets a photorefractive gain $\Gamma=0.7 \mathrm{~cm}^{-1}$ that exceeds typical gains in GaAs and InP by more than $50 \%(13)$.

The analysis of the gain dependence with the grating period will provide us information about the effective density of traps involved in the photorefractive effect. To always achieve the largest possible gain, we choose an input intensity that saturates the photorefractive gain. Considering a single deep level and two possible charge carriers, the gain expression is $(16)$ :

$$
|\Gamma|=\frac{2 \pi n_{0}^{3}\left|r_{41}\right|}{\lambda \cos \theta} \frac{k_{B} T}{e} \frac{k \xi_{0} k^{2}+b-c \mid}{\left(k^{2}+\frac{k^{4}}{k_{0}^{2}}+b+c\right)}
$$

with:

$$
k_{0}^{2}=\frac{e^{2}}{\varepsilon k_{B} T} N_{e f f} \quad \xi_{0}=\frac{\left(\alpha_{n}-\alpha_{p}\right)}{\left(\alpha_{n}+\alpha_{p}\right)} \quad b=\frac{\left(\alpha_{n} \kappa_{p}^{2}\right)}{\left(\alpha_{n}+\alpha_{p}\right)} \quad c=\frac{\left(\alpha_{p} \kappa_{n}^{2}\right)}{\left(\alpha_{n}+\alpha_{p}\right)}
$$

$\alpha_{n}$ and $\alpha_{p}$ are the absorption contributions that come from electron and hole generation respectively. $\kappa_{n}$ and $\kappa_{p}$ are the inverse of electron and hole diffusion lengths. $\mathrm{k}$ represents the grating wave number and $\mathrm{N}_{\text {eff }}$ is the effective trap density $\left(\mathrm{N}_{\text {eff }}=\mathrm{N}^{+} . \mathrm{N}^{0} /\left[\mathrm{N}^{0}+\mathrm{N}^{+}\right]\right.$where $\mathrm{N}^{+}$and $\mathrm{N}^{0}$ are the level concentrations in the ionized and neutral states respectively). Note that here we assume that electrons are the majority carrier for the photorefractive effect. In the opposite case, index $n$ and $p$ have to be exchanged. At present we do not know whether the dominant photorefractive carrier in this sample is electron or hole.

As now routinely done, the photorefractive gain $\Gamma$ is deduced from the measurement of the amplification (attenuation) of one of the interfering beam in presence of the other. Plotting our experimental data as $\mathrm{k} / \Gamma$ versus $\mathrm{k}^{2}$ (fig.9) permits the determination of the characteristic photorefractive parameters $(15)$. For large values of $k^{2}$, coefficients $b$ 
and $\mathrm{c}$ are negligible and we get a straight line. Its slope and intercept at the origin give $\left(\mathrm{r}_{4} 1 \xi_{0} \mathrm{k}_{0}\right)^{-1}$ and $\left(\mathrm{r} 41 \xi_{0}\right)^{-1}$. A departure from this straight line is visible at small values of $\mathrm{k}^{2}$. This indicates the influence of the carrier diffusion lengths. A full fit of the experimental data with the complete gain expression, i.e., without neglecting $b$ and c, is now carried out using the previously determined quantities as starting parameters. We obtain:

$$
\begin{aligned}
& \xi_{0}=0.62+/-0.06 \\
& \mathrm{r} 41=(5.5+/-0.5) \mathrm{pm} \mathrm{V}^{-1} \\
& \mathrm{k}_{0}^{2}=(300+/-30) \mu \mathrm{m}^{-2} \\
& \mathrm{~b}=(3.6+/-1.5) \mu \mathrm{m}^{-2} \\
& \mathrm{c} \approx 0
\end{aligned}
$$

Also known is the previously measured absorption: $\alpha=(2.16+/-0.06) \mathrm{cm}^{-1}$.

These results call on the following remarks. First, the $\xi_{0}$ value indicates an electronhole competition that is larger than previously seen in different samples $(5)$. Nevertheless, the photorefractive gain has an appreciable value. Second, coefficient b permits an estimation of the mobility-life time product of the minority carrier for the photorefractive effect. Assuming, as in ref. 17, that electrons are the majority photocarriers for the photorefractive effect, one gets $\kappa_{\mathrm{n}}^{2}<<\kappa^{2}$ and derives for the minority carriers (holes in this case):

$$
\mu \tau=9.10^{-8} \mathrm{~cm}^{2} \mathrm{~V}^{-1} \text {. }
$$

This value is about one order of magnitude smaller than what we determined by photoconductivity measurements that were giving the mobility-life time product for the majority carrier (assumed to be electrons).

From $\mathrm{k}_{0}^{2}$, we inferre the effective deep level density that contributes to the photorefractive effect, $\mathrm{N}_{\mathrm{eff}}=4.3 \times 1015 \mathrm{~cm}^{-3}$. As discussed in ref.2, in the low illumination quasicontinuous regime as here considered, the presence of shallow traps does not change the steady state photorefractive effect. Thus, these CW photorefractive experiments do not feel the influence of the multiple shallow traps we saw in our spectroscopic studies. It is believed that the efficient deep level for the photorefractive 
effect might be the P7 level identified in table 1. Considering the results presented in section 3, it appears that this density might be determined by other defects than vanadium in the CdTe $1 \mathrm{H}$ crystal $(7,8,12)$.

We also conducted similar experiments at $\lambda=1.32 \mu \mathrm{m}$. First, as pointed out in section 3 and in 4.1, we observed a nearly five time drop of the photoconductivity. However, for the incident illuminations used, the dark conductivity is still overpassed. Second, the magnitude of the photorefractive gain was very small $\left(\Gamma=0.06 \mathrm{~cm}^{-1}\right.$ at $\Lambda=1.5 \mu \mathrm{m}$ ), impeding any reliable measurement versus fringe spacing. One only estimates an electron-hole competition factor $\xi_{0}=0.1$ that corresponds to nearly balanced absorption coefficients, $\alpha_{n}=\alpha_{p}$.

It is clear that a contribution from multiple defects might produce such a reduction of the photorefractive gain. However, an important point we noticed, is that the photorefractive gain reverses its sign when changing the wavelength from 1.06 to 1.32 $\mu \mathrm{m}$. This indicates a change in the nature of the photorefractive majority carrier. This result supports the approach we used for describing the photorefractive effect observed, i.e., a single deep level from which both electrons and holes are photoionized. Such a reverse in the sign of the photorefractive gain as the incident wavelength was varied, was not noticed for the crystals analyzed in ref. 17.

\section{Conclusion.}

We have here reported the first results of our joint research program for growth, caracterization and optimization of CdTe for photorefractive applications.

High resistivity samples have been synthetized and first characterizations including spectroscopic and photorefractive ones have been made. For sample $1 \mathrm{H}$ ( low vanadium concentration), 7 levels have been seen and their activation energy determined. The investigation of trapping levels deeper than $0.6 \mathrm{eV}$ will be of particular interest for photorefractivity. This will be done after achieving a slight modification of the detection procedure of the current transients. This sample exhibits a 
fair photorefractive effect at $\lambda=1.06 \mu \mathrm{m}$ with nevertheless a non negligible electronhole competition. At $\lambda=1.32 \mu \mathrm{m}$, the effect has a reverse sign and the competition is very strong leading to a nearly full compensation. The photoconductivity spectrum of sample 6B (more heavily vanadium doped) already shows that this crystal will better behave at longer wavelengths. Similar characterizations are now in progress with this crystal and samples with other specifications are currently prepared. 


\section{References}

1_G. Le Saux, A. Brun, IEEE, J. Q. Elect. QE 23, 1680, 1987

2_G. Pauliat, G. Roosen, J. Opt. Soc. Am. B, 7, 2259, 1990

3_R.B. Bylsma, P.M. Bridenbaugh, D.H. Olson, A.M. Glass, Appl. Phys. Lett., 51, 889,1987

4_J.M. Langer, C. Delerue, M. Lanoo, H. Heinrich, Phys. Rev. B, 38, 7723, 1988

5_A. Partovi, J. Millerd, E.M. Garmire, M. Ziari, W.H. Steier, S.B. Trivedi, M.B.

Klein, Appl. Phys. Lett., 57, 846, 1990

6_ J.M. Dance, private communication

7_ C. Scharager, J.C. Muller, R. Stuck, P. Siffert, Phys. Stat. Sol. (a), 31, 247, 1975

8_ M. Samini, B. Biglari, M. Hage-Ali, P. Siffert, J. Cryst. Grow., 72, 213, 1985

9_ P.I. Babii, N.P. Gavaleshko, Yu.P. Gnatenko, P.A. Skubenko, V.I. Oleinik, Sov. Phys. Semicond., 12, 1310, 1979

10_ J.C. Balland, J.P. Zielinger, C. Noguet, M. Tapiero, J. Phys. D: Appl. Phys., 19, 57, 1986; J.C. Balland, J.P. Zielinger, M. Tapiero, J.G. Gross, C. Noguet, J. Phys. D: Appl. Phys., 19, 71, 1986; M. Tapiero, N. Benjelloun, J.P. Zielinger, S. El Hamd, C. Noguet, J. Appl. Phys., 64, 4006, 1988; N. Benjelloun, M. Tapiero, J.P. Zielinger, J.C. Launay, F. Marsaud, J. Appl. Phys., 64, 4013, 1988;

11_N.V. Agrinskaya, O.A. Matveev, A.I. Terent'ev, V.V. Shasshkova, Sov. Phys. Semicond., 23, 274, 1989.

12_A.J. Strauss, Rev. Phys. Appl., 12, 167, 1977.

13_Ph. Delaye, P.U. Halter, G. Roosen, J. Opt.Soc.Am.B, 7, 2268, 1990.

14_J.C. Fabre, J.M.C. Jonathan, G. Roosen, J. Opt.Soc.Am.B, 5, 1730, 1988.

15_M.B. Klein, G.C. Valley, J.Appl. Phys. 57, 4901, 1985.

16_F.P. Strohkendl, J.M.C. Jonathan, R.W. Hellwarth, Opt. Lett. 11, 312, 1986.

17_M. Ziari, W.H. Steier, M.B. Klein, S. Trivedi, 3rd Topical Meeting on Photefractive Materials,Beverly, 1991 Technical Digest 14, 159. 
Table 1

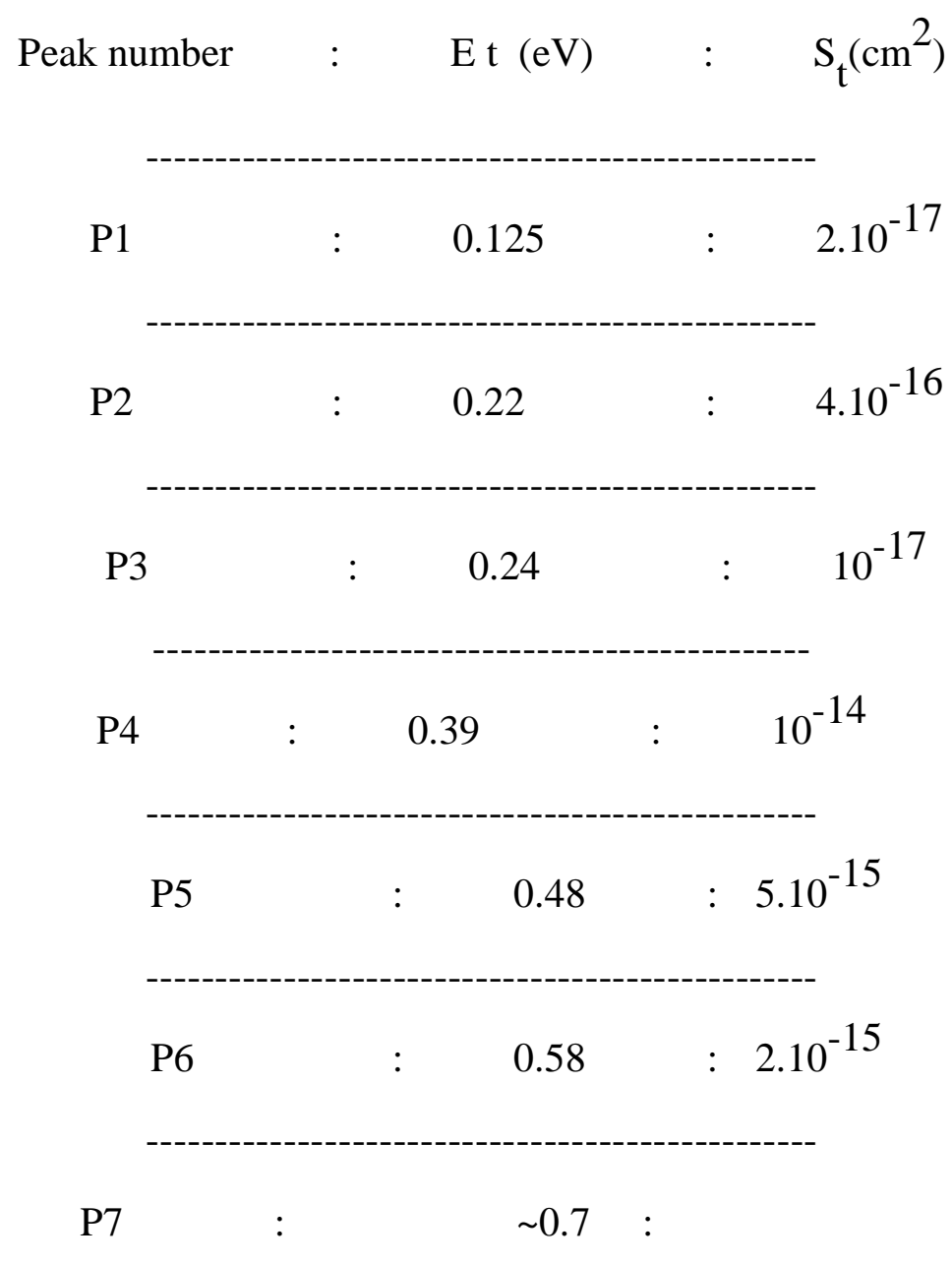




\section{Figure captions}

Figure 1. Typical shape of the photocurrent transient induced by a square light pulse. During illumination the current rises up to a constant value $i(0)$. The light is interrupted at time $\mathrm{t}=0 ; \mathrm{t}_{0}, \mathrm{t}_{1}, \mathrm{t}_{2}, \mathrm{t}_{3}$ are fixed delay times.

Figure 2. Illustration of the principles of data processing (double-gate method). The left hand curve shows the transient at various temperatures; $t_{1}$ and $t_{2}$ are fixed delay times. The right hand curve shows the temperature dependance of the differential signal $\mathrm{n}_{\mathrm{t}}\left(\mathrm{t}_{1}\right)-\mathrm{n}_{\mathrm{t}}\left(\mathrm{t}_{2}\right)$.

Figure 3. Normalized photoconductivity spectrum; curve 1, weakly doped sample (1H); curve 2, strongly doped sample (6B).

Figure 4. Curve 1: Photoconductivity versus temperature

Curve 2: Typical double gate spectrum

Figure 5. Typical normalized double gate (curve 1) and four gate (curve 2) spectra.

Figure 6. Typical Arrhenius plot for peak number $3\left(E_{t}=0.24 \mathrm{eV}\right.$ and $S_{t}=10^{-17}$ $\left.\mathrm{cm}^{2}\right)$

Figure 7. Absorption spectrum of sample $1 \mathrm{H}$.

Figure 8 . Photorefractive gain of sample $1 \mathrm{H}$ versus incident illumination $(\Lambda=0.9$ $\mu \mathrm{m})$ showing a saturation of the gain for incident powers as low as $20 \mathrm{~mW} . \mathrm{cm}^{-2}$. Figure 9. Experimental $\Gamma / \mathrm{k}$ versus $\mathrm{k}^{2}$ plot leading to the determination of the photorefractive parameters of crystal $1 \mathrm{H}$. This plot was drawn with $\Gamma$ in $\mathrm{cm}^{-1}$ and $\mathrm{k}$ in $\mu \mathrm{m}^{-1}$.The dotted straight line is the fit obtained for large grating wave numbers while the full line curve represents the best fit with the whole gain expression. 


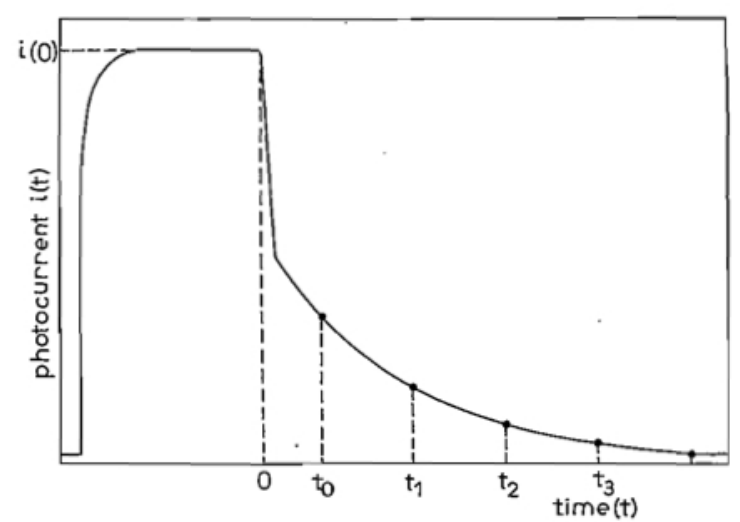

Figure 1

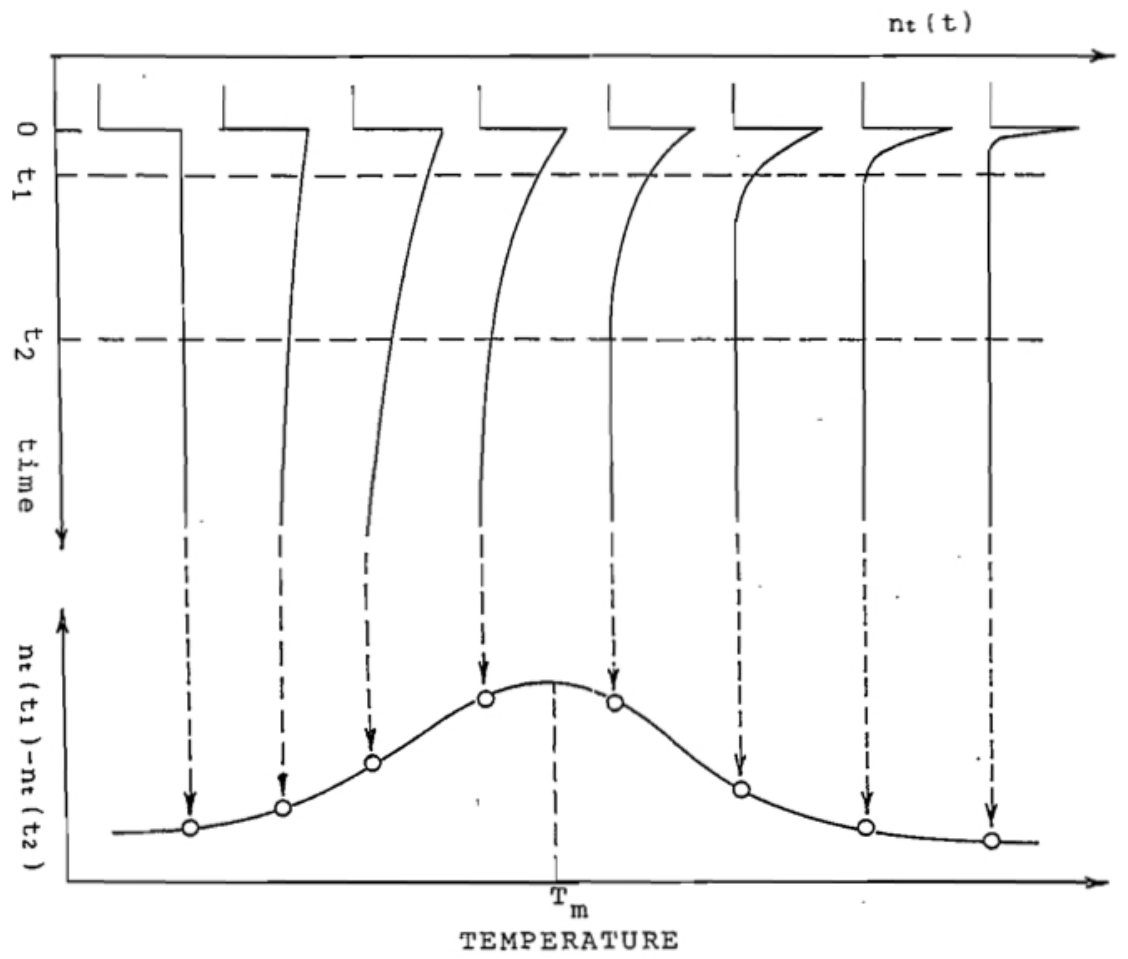

Figure 2

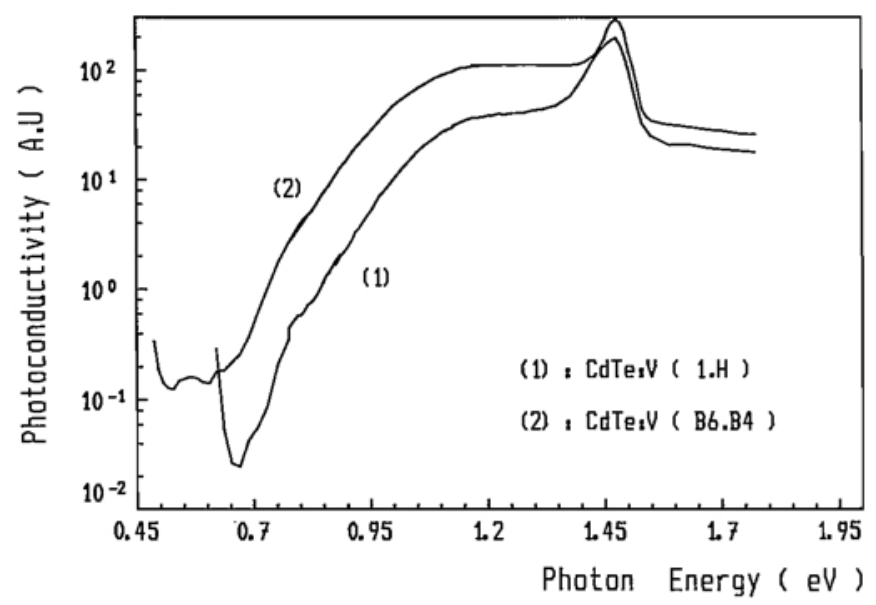

Figure 3 


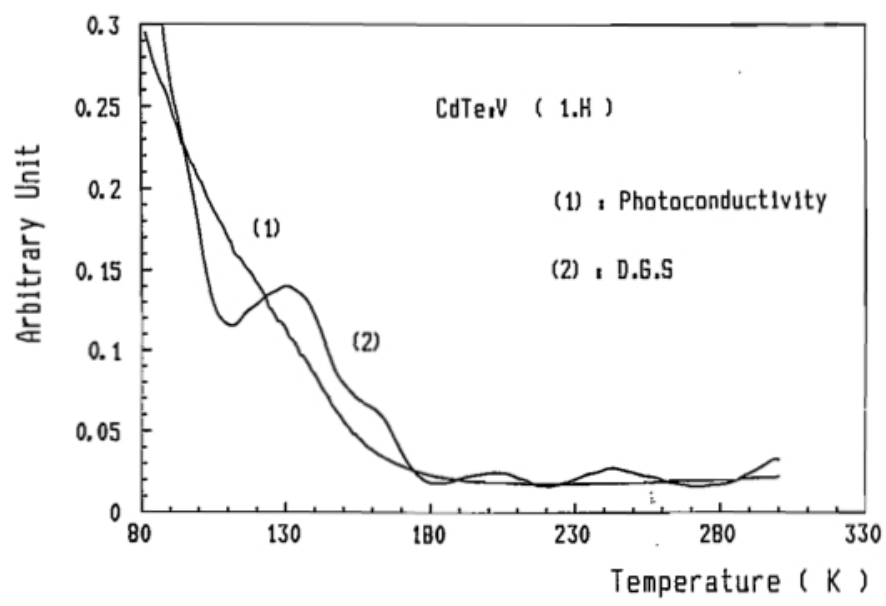

Figure 4

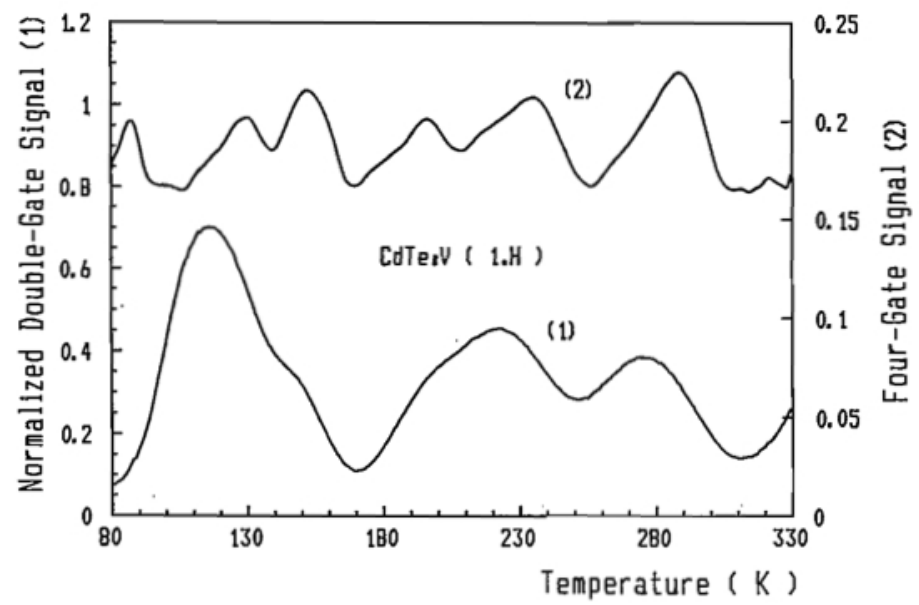

Figure 5

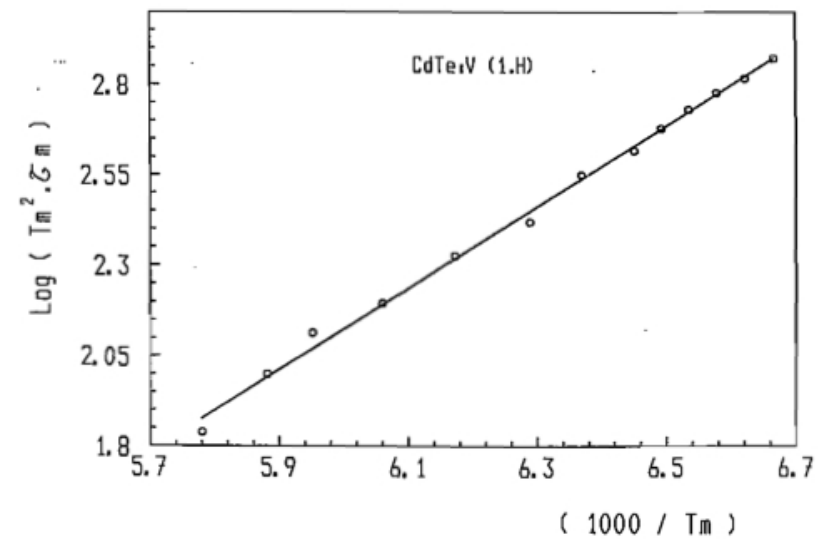

Figure 6 


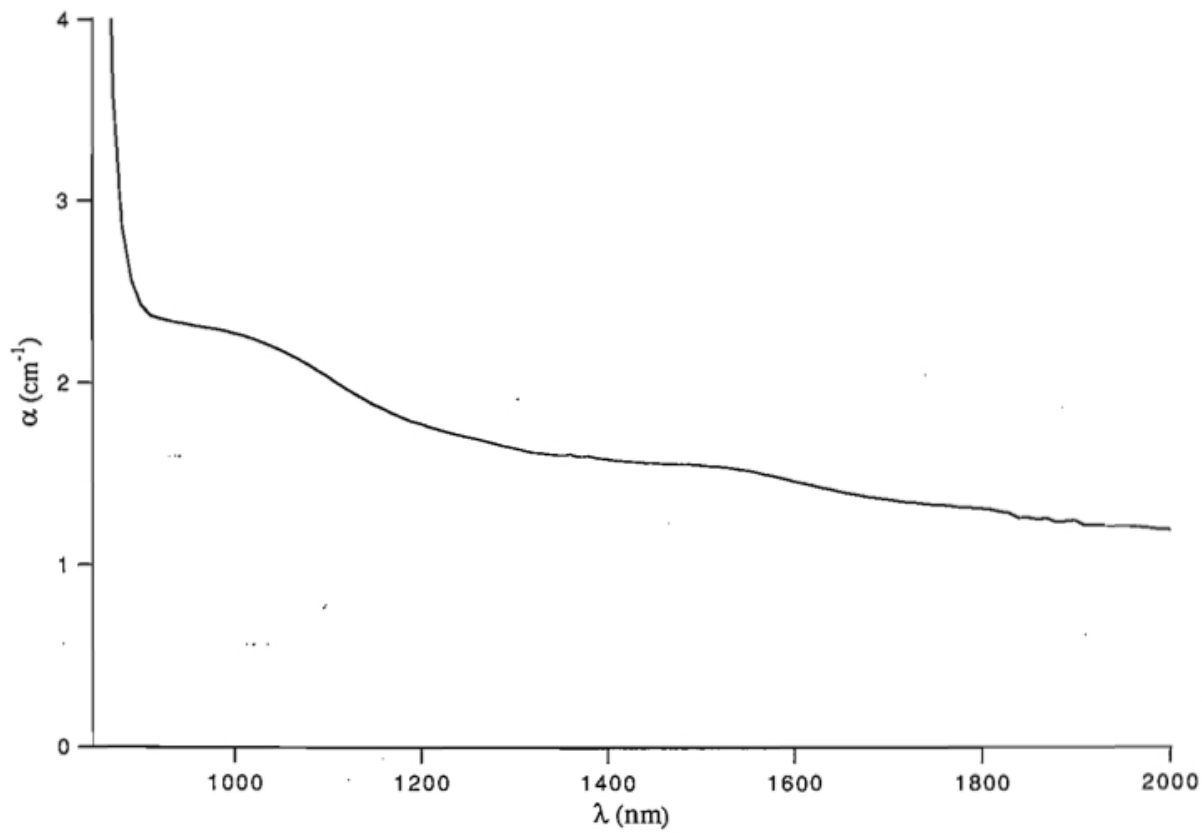

Figure 7

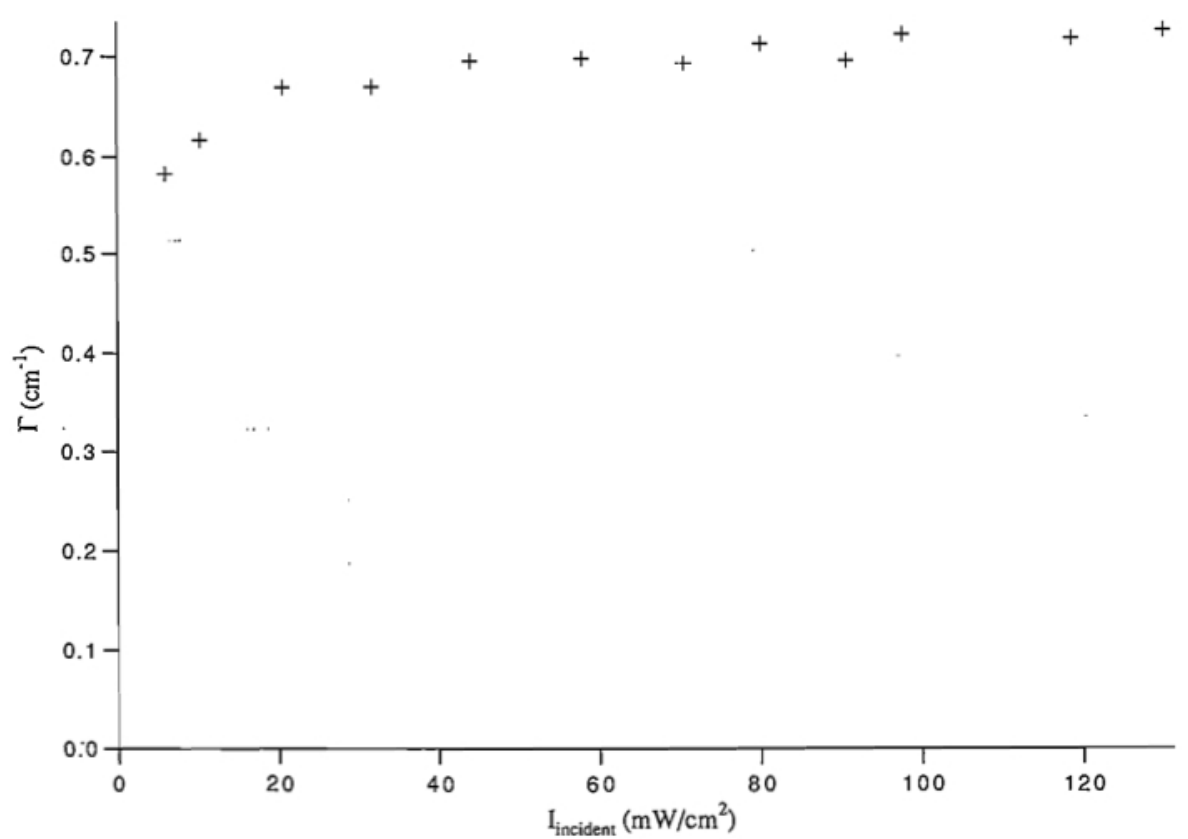

Figure 8 


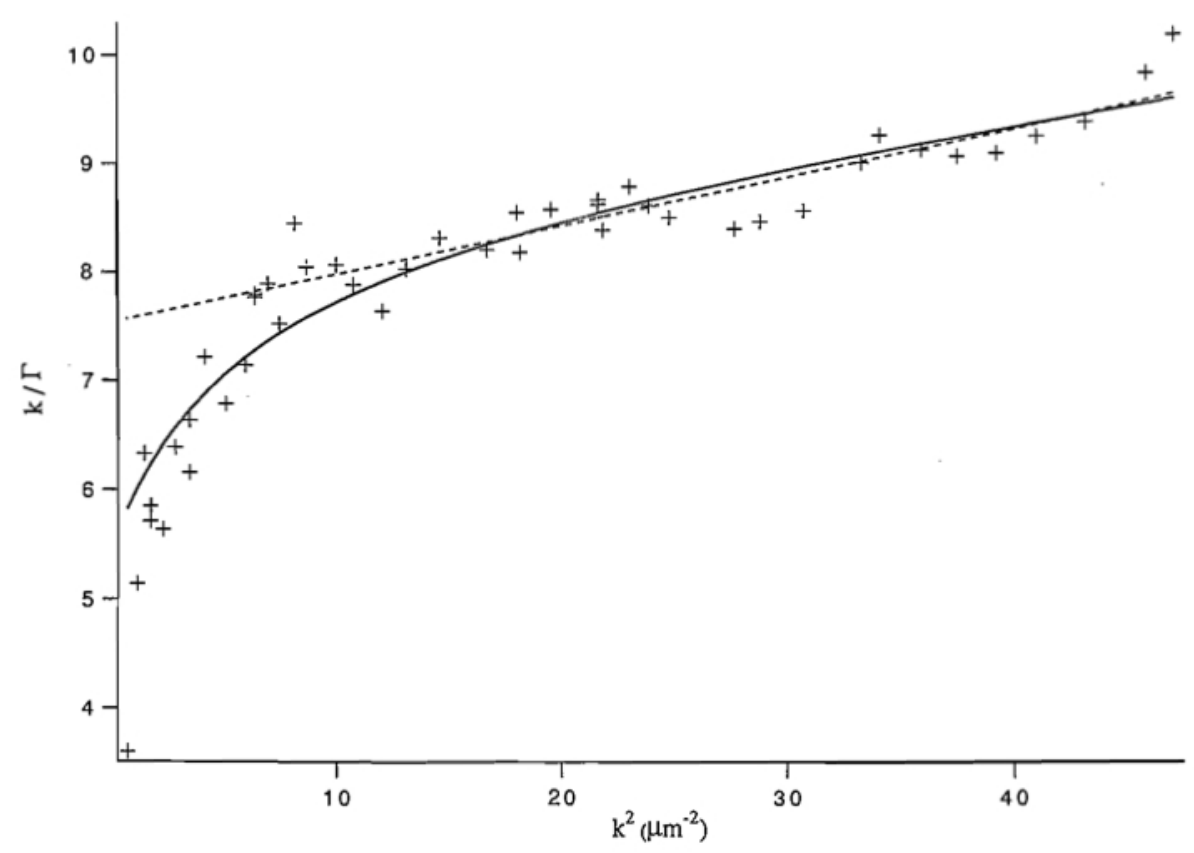

Figure 9 